\title{
Brugada phenocopy induced by tricyclic antidepressant poisoning
}

\author{
Ellepola $\mathrm{KD}^{1}$, Jayasinghe $\mathbf{I K}^{1}$ \\ Journal of the Ceylon College of Physicians, 2021, 52, 56-58
}

\begin{abstract}
Tricyclic antidepressants (TCAs) are widely used to treat major depressive disorders. TCAs act mainly by inhibiting presynaptic reuptake of norepinephrine and serotonin in the central nervous system. Overdose of TCAs can result in devastating effects by antagonizing peripheral alpha-adrenergic, histaminic, muscarinic, and central serotonin receptors leading to a variety of adverse effects including anticholinergic toxicity and cardiac toxicity. In addition, blockade of fast sodium channels in myocardial cells leads to slowing of action potential, triggering conduction blocks and bradycardia.

Brugada phenocopy with electrocardiographic (ECG) evidence of Brugada syndrome can be found in acquired clinical circumstances following metabolic derangements and myocardial ischaemia. A few cases of Brugada phenocopy following TCA intoxication has been reported in the literature so far. We report a case of 56-year-old female who presented with acute intoxication of amitriptyline with ECG evidence of Brugada syndrome that ultimately led to pulseless ventricular tachycardia.
\end{abstract}

Key words: TCA poisoning, Brugada phenocopy

\section{Introduction}

Tricyclic antidepressants (TCAs) are widely used as pharmacotherapy for major depressive disorders, migraine prophylaxis, neuralgic pain, obsessivecompulsive disorders, insomnia and nocturnal enuresis in children.
TCAs act mainly by inhibiting presynaptic reuptake of norepinephrine and serotonin in the central nervous system. They also act on post-synaptic alpha cholinergic, muscarinic, and histaminergic receptors exerting a competitive antagonistic effect. ${ }^{1}$ TCA toxicity exerts antagonistic effects on peripheral alphaadrenergic, histaminic, muscarinic, and central serotonin receptors leading to anticholinergic and cardiac toxicity. Slowing of action potential leading to heart blocks and bradycardia is caused by concomitant blockade of fast sodium channels in myocardial cells. ${ }^{2}$

Brugada phenocopy contains ECG evidence similar to Brugada syndrome and can be provoked by various clinical circumstances such as acquired sodium channel dysfunction or myocardial ischaemia. ${ }^{2}$ There are only a few published case reports on Brugada like ECG patterns following TCA intoxication.

\section{Case presentation}

A 56-year-old woman with a history of depression, presented with deliberate self-ingestion of 20 tablets of amitriptyline two hours prior to admission. On admission, she was drowsy with a Glasgow coma scale (GCS) of 13/15. She had a tachycardia of 102 bpm and blood pressure of $140 / 90 \mathrm{mmHg}$. Both pupils were normal in size and reactive. Initial ECG revealed sinus tachycardia with narrow QRS complexes.

Activated charcoal $50 \mathrm{~g}$ was given for gastric decontamination. Her condition deteriorated after admission and the level of consciousness deteriorated to a GCS of 10/15. Both pupils became dilated. Tachycardia worsened to $120 \mathrm{bpm}$, with a regular rhythm. The blood pressure was recorded as $120 / 70 \mathrm{mmHg}$.

${ }^{1}$ National Hospital, Kandy, Sri Lanka.

Correspondence:KDE, e-mail: kithminiel@gmail.com

https://orcid.org/0000-0002-7800-8710

Received 04 April 2021, accepted 18 May 2021.

This is an open-access article distributed under the terms of the Creative Commons Attribution License, which permits unrestricted use, distribution, and reproduction in any medium, provided the original author and source are credited. 


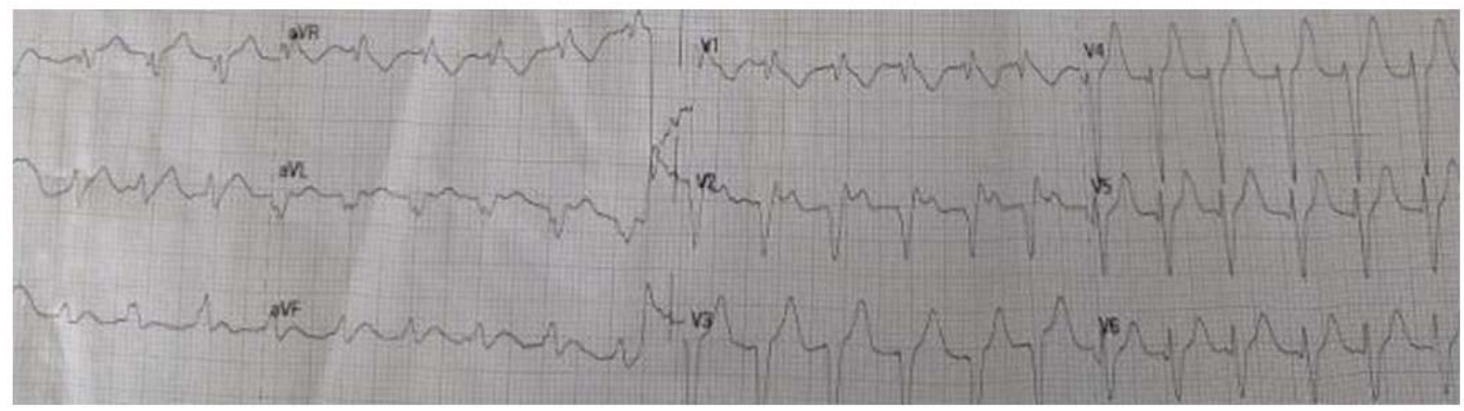

Figure 1. ECG showing saddle shaped ST elevation in lead $\mathrm{V}_{2}$ suggestive of type 2 Brugada pattern and other cardiac toxic features of TCA overdose.

ECG which was taken at this point revealed broad QRS complex (120ms) tachycardia. Saddleback shaped ST elevation was noted in lead $\mathrm{V}_{2}$ compatible with type 2 Brugada pattern. There were also deep, slurred S wave in leads I and $\mathrm{aVL}, \mathrm{R} / \mathrm{S}$ ratio $>0.7$ in lead $\mathrm{aVR}$ and terminal $\mathrm{R}$ wave $>3 \mathrm{~mm}$ in aVR which were highly sug-gestive of cardiac toxicity of TCA overdose (Figure 1).

Intravenous sodium bicarbonate $100 \mathrm{~mL}$ was given, followed by $100 \mathrm{~mL}$ in $400 \mathrm{~mL}$ of $5 \%$ dextrose infusion with continuous cardiac monitoring. Two hours later, the patient became unresponsive with a GCS of $3 / 15$. Pulse was not palpable and blood pressure was not recordable. Heart rate at cardiac monitor was 200 bpm with morphology being a ventricular tachycardia. A diagnosis of pulseless ventricular tachycardia was made.

Cardiopulmonary resuscitation (CPR) was commenced followed by $150 \mathrm{~J}$ of direct cardioversion. Sodium bicarbonate $100 \mathrm{~mL}$ was given intravenously. She was intubated with continuation of CPR. The cardiac rhythm was reverted to sinus rhythm with a heart rate $116 \mathrm{bpm}$. Sodium bicarbonate infusion was commenced at a rate of $50 \mathrm{~mL} /$ hour in the aim of maintaining urinary $\mathrm{pH} 7.4-7.5$ and arterial $\mathrm{pH}$ 7.457.55. Serial arterial blood gas estimations and cardiac monitoring was continued. She made a complete recovery within next few days. Her subsequent ECGs did not show any abnormalities.

\section{Discussion}

The Brugada syndrome is defined as an autosomal dominant channelopathy, produced by the dysfunction of a cardiac channel participating in the action potential. The electrical disorder is usually primary, without concomitant underlying structural heart disease.
Interestingly, ECG findings mimicking Brugada syndrome can be found even in the absence of congenital dysfunction of ion channels. This so called acquired Brugada syndrome is currently defined as Brugada phenocopies. Brugada phenocopies are usually induced by reversible clinical conditions and are related to metabolic imbalances, ischemia, and pulmonary embolism. The pathogenesis by which the phenotype is induced is due to an imbalance between ion currents during the phase 1 of the action potential or by conduction delays in the anterior myocardial wall. ${ }^{3}$

TCAs cause blockade of fast sodium channels, alpha 1 adrenergic, muscarinic acetylcholine, histamine 1 and CNS gamma-aminobutyric acid type A receptors thus causing neurologic, cardiac, and anticholinergic toxicity in a setting of an overdose. ${ }^{2}$ TCAs act as class la anti-arrhythmic drugs in an over-dose and affect the cardiac action potential, lengthening both depolarization and repolarization phases, widening the QRS complex, prolonging the QT interval or causing a Brugada phenocopy. In this setting, Brugada phenocopy is caused by transient sodium channel dysfunction. It poses a highly increased risk for malignant ventricular arrhythmias and death. ${ }^{4}$

There are a few case reports of Brugada phenocopy due to TCA induced cardiac toxicity. ECG evidence with Brugada type 1 pattern with ultimate marked QRS widening, ventricular fibrillation and cardiac arrests following therapeutic dosing and overdose of TCA has been reported so far. ${ }^{4,5}$

Our patient presented with ECG manifestations compatible with type 2 Brugada pattern following TCA overdose as a form of cardiac toxicity. Her ECGs also revealed deep, slurred $S$ wave in leads I and $\mathrm{AVL}, \mathrm{R} / \mathrm{S}$ ratio $>0.7$ in lead aVR and terminal $R$ wave $>3 \mathrm{~mm}$ in aVR which were highly suggestive of cardiac toxicity in a TCA overdose (Figure 2). 


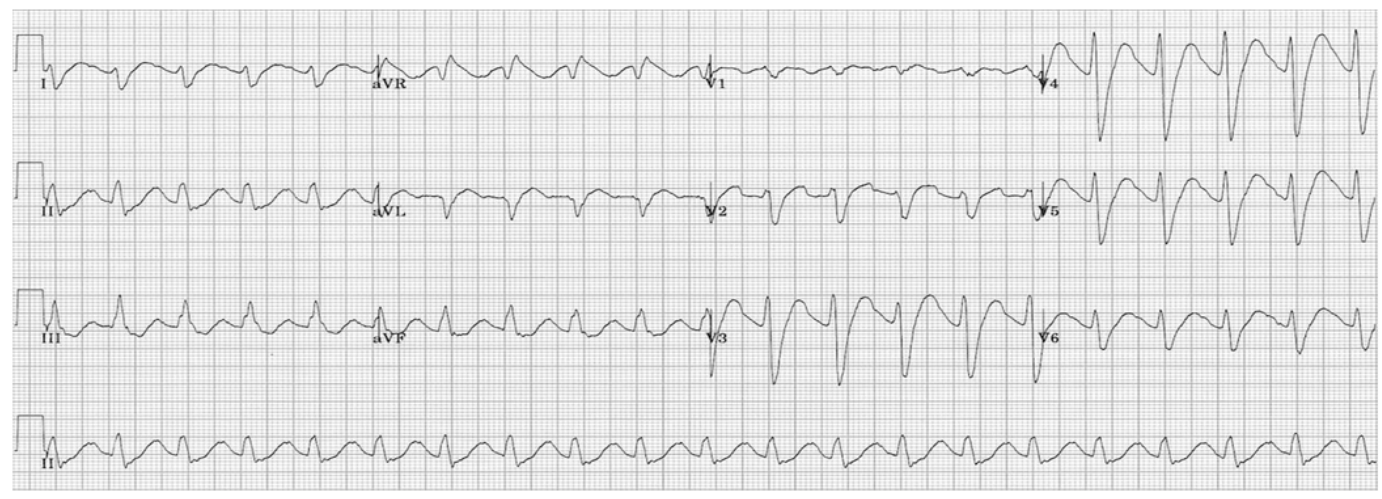

Figure 2. ECG revealing cardiac toxic features of TCA overdose with $S$ wave in 1 and aVL, terminal $R$ wave in aVR and QRS widening; ECG obtained from the article 'Tricyclic overdose' URL: https://litfl.com/tricyclic-overdose-sodium-channel-blockertoxicity/].

Her clinical condition was complicated with pulseless ventricular tachycardia requiring advanced life support and defibrillation. She was subsequently managed with serum alkalization with intravenous sodium bicarbonate, which is the standard treatment form for TCA poisoning.

\section{Conclusion}

This case report highlights the importance of knowing the concurrence of Brugada phenocopy as an ECG manifestation of cardiac toxicity of TCA poisoning as it can lead to fatal arrhythmias and sudden cardiac deaths. Early identification and prompt management of this unmasked phenotype can save many lives.

\section{Consent}

Informed written consent was obtained from the patient for publication of this case report.

\section{Author contributions}

EKI: Wrote the case report, drafted, and edited the manuscript. JIK: Contributed to writing, edited the manuscript.

\section{Conflicts of interests}

The authors declare that there are no conflicts of interest.

\section{References}

1. Gillman PK. Tricyclic antidepressant pharmacology and therapeutic drug interactions updated. British Journal of Pharmacology 2007; 151(6): 737-48. doi:10.1038/ sj.bjp.0707253

2. Otero D, Petrovic M, Liao SL. Brugada Phenocopy: A Case of Incessant Ventricular Tachycardia in a Patient with Tricyclic Antidepressant Overdose. Methodist Debakey Cardiovascul. J. 2020; 16(3): 245-8. doi: 10.14797/mdcj16-3-245.

3. De Oliveira Neto, NR, de Oliveira WS, Mastrocola F, Sacilotto L. Brugada phenocopy: Mechanisms, diagnosis, and implications. J. Electrocardiol. 2019; 55: 45-50. doi:10.1016/ j.jelectrocard.2019.04.017

4. Meert A, Vermeersch N, Beckers R, Hoste W, Brugada P, Hubloue, I. Brugada-like ECG pattern induced by tricyclic antidepressants. Eur J Emerg Med. 2010; 17(6), 325-7. doi:10.1097/mej.0b013e328334a98f

5. Yap YG, Behr ER, Camm AJ. Drug-induced Brugada syndrome. Europace 2009; 11(8): 989-94. doi: 10.1093/ europace/eup114. Epub 2009 May 29. 